\title{
Risques associés à l'utilisation de la méthode des éléments finis pour la géotechnique
}

\section{R.B.J. BRINKGREVE'1, 2}

R.P.B. WITASSE ${ }^{1}$

${ }^{1}$ Plaxis bv, Delftechpark 53

PO Box 572

2600 AN Delft

The Netherlands

${ }^{2}$ Delft University of Technology

Section Geo-Engineering

PO Box 5048

2600 GA Delft

The Netherlands
La méthode des éléments finis a considérablement évolué au cours des vingt dernières années pour devenir aujourd'hui un outil essentiel pour le dimensionnement et l'analyse en ingénierie géotechnique. Pourtant,

l'exactitude et la pertinence des calculs en éléments finis dépendent principalement de la capacité de l'utilisateur à parfaitement comprendre et maîtriser les possibilités et les limitations des méthodes et modèles employés. Cet article a pour but de dresser un inventaire des limitations et des principaux risques associés à l'utilisation de cet outil numérique particulier afin d'en accroître la connaissance et d'améliorer la qualité des résultats fournis.

Mots-clés : analyse éléments finis, risques, limitations, exactitude, modélisation numérique.

\section{Risks related to geotechnical finite element analysis}

The finite element method has evolved as an essential tool in geotechnical engineering, analysis and design over the last 20 years. However, the accuracy and therefore the relevance of finite element calculations mostly depends on the ability of the user to clearly understand and properly deal with the possibilities and limitations of the various models and methods being used. This publication intends to create awareness for those risks with the purpose to increase the knowledge of the limitations and to improve the quality of the results.

Key words: finite element analysis, risks, accuracy, limitations, numerical modeling. 


\section{Introduction}

Since the early nineties of the previous century, the finite element method has evolved as an essential tool in geotechnical engineering, analysis and design. The advantages of the method over conventional design methods are quite clear. The finite element method can deal with different types of design issues: settlements, deformations, stability, structural forces, but also issues related to groundwater flow or temperature. Geometries and construction stages can be modelled realistically and there is no pre-assumption of a particular mechanism.

In comparison with conventional analysis, the finite element method requires more data to be specified, but user-friendly software packages exist that enable a relatively fast modelling procedure. Despite the userfriendliness, there are some traps and pitfalls related with the limitations of the numerical methods and the models being used. These limitations are not always recognized; neither by the often young project engineers who operate the software, nor by their managers who control their work. This may lead to risks related to the use of the finite element method for geotechnical analysis and design. This publication intends to create awareness for those risks with the purpose to increase the knowledge of the limitations and to improve the quality of results.

With the increasing computer power it is now feasible to perform fully three-dimensional (3D) finite element calculations for complex situations, but most calculations are still two-dimensional (2D). The more finite elements are used, the more accurate the results might be in principle, but the more time is required to perform the calculations. Setting up a finite element model requires decisions about the type of analysis to be used, the detail and extend of the geometries to be included in the model, the position of the model boundaries, the soil constitutive models to be used, the fineness of the finite element mesh, the generation of initial conditions, the definition of construction stages, the types of calculations to be used and finally the interpretation and translation of the results into geotechnical design aspects. Each of the decisions that are made requires knowledge about the possibilities and limitations of the corresponding modelling features. This publication describes a number of such limitations, mostly related to the mechanical behaviour of soil structures (deformation and stability). Thereby, the publication is structured according to the various modelling categories as described above. In the end a visual checklist is proposed as an efficient method to check finite element modelling issues. The publication ends with the major conclusions and a reference list for further reading.

\section{2}

\section{Geometric modelling}

The first decision that needs to be made to set up a finite element model is the type of analysis to be used. Distinction is made between a 2D plane strain model, a 2D axi-symmetric model or a 3D model. The position of the boundaries is also part of the decision process. In the case the situation is symmetric, it may be decided to model only one symmetric half, while appropriate boundary conditions are used reflecting the symmetry. Other boundaries should be sufficiently far away such that the boundary conditions do not influence the results. Also decisions need to be made about the modelling of structures; using volume elements or using special structural elements. When structures are being modelled, soil-structure interaction also needs to be properly accounted for, and special interface elements or contact elements can be used for this purpose. In the following some traps and pitfalls related to the type of analysis, the selection of model boundaries and special elements are described.

\section{7.}

\section{Type of analysis}

A plane strain model is a two-dimensional model in which the strain perpendicular to the modelling plane is zero. Note that the stress is generally nonzero and it can change as a result of loading. Typical situations that could be modelled with a plane strain model involve structures where one dimension is long compared to the other dimensions, such as embankments, slopes, strip foundations, retained excavations and tunnels. However, when the conditions change along the long direction, it should be considered whether a plane strain model is still appropriate or whether a 3D model should be used (Fig. 1). In case a plane strain model us used anyway, it should be considered whether the results are optimistic or conservative.

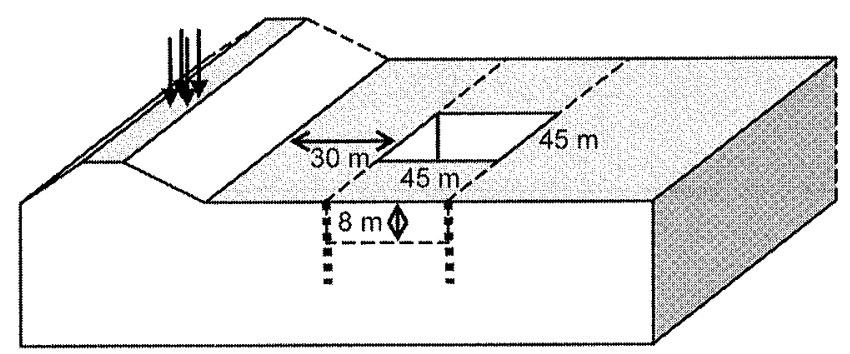

H16.1. Non plane strain situation example.

An axi-symmetric model is a two-dimensional model which is symmetric around its central axis. Deformations and stresses perpendicular to the axis of symmetry apply to any radial direction. Typical situations that could be modelled with an axi-symmetric model involve structures that are circular, such as circular footings, piles, oil tank foundations and suction caissons. However, as soon as the situation involves conditions perpendicular to the central axis which are not radial, it should be considered whether an axi-symmetric model is still appropriate or whether a 3D model should be used (Fig. 2). In case an axi-symmetric model is used anyway, it should be considered whether the results are optimistic or conservative. 

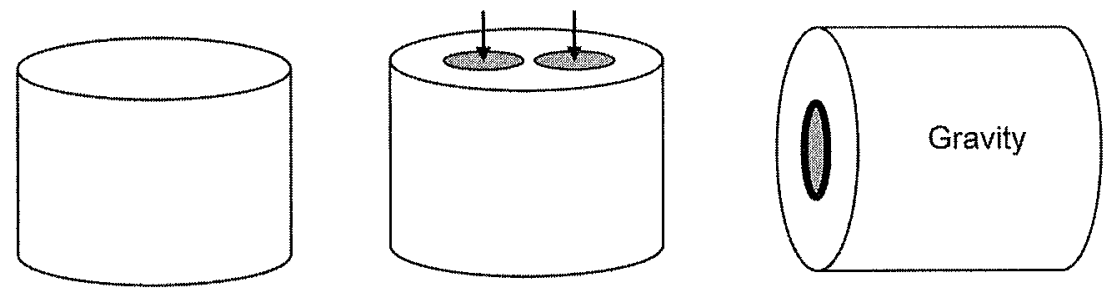

F6. 2 Non axi-symmetric situation examples.

In the case a situation is neither plane strain nor axi-symmetric, it should be considered whether a 3D model is really required. Although 3D calculations are quite feasible nowadays, they are more complex and time-consuming than 2D calculations. Moreover, 3D finite element meshes are generally coarser than 2D meshes due to their relatively high computational cost which could possibly lead to less accurate results.

Another interesting case is the modelling of structures involving one or more rows of piles, such as pile foundations or quay walls. When using a plane strain model, the piles could be modelled by means of beam elements (Fig. 3). However, it should be noticed that the beam elements in a plane strain model actually represent plates in the out-of-plane direction. Especially when the piles are subjected to lateral loading or lateral soil movement, this way of modelling may become highly unrealistic. In reality, lateral soil movement can be different than the lateral pile movement, but a plane strain model cannot cope with this.
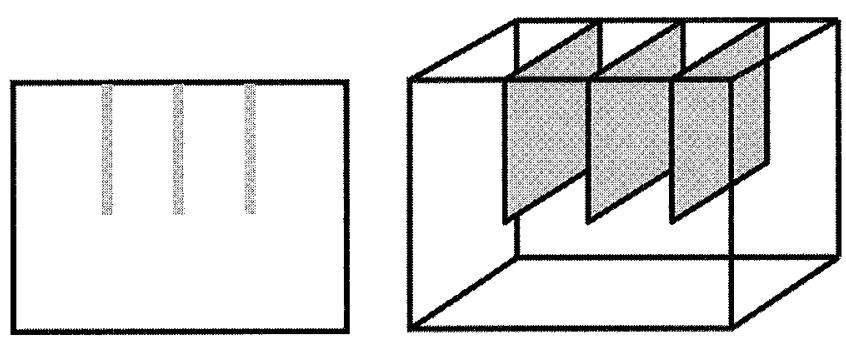

Fic. 3 Modelling rows of piles by means of beam/ plate elements.

\section{Model boundaries}

Model boundaries should be chosen sufficiently far from the part of the model where the action takes place. This applies to the vertical sides of the model as well as the bottom boundary. The main criterion is that the model boundaries should not influence the analysis results. This requires that the dominant "mechanism" should fit in the model. The type of analysis (drained or undrained deformation analysis, stability analysis, dynamic analysis, etc.) may influence the decision where to define the model boundaries (Fig. 4).

It is also worthwhile mentioning that in dynamic calculations special boundary conditions should be used to damp waves that reach the model boundaries. However, these boundary conditions are never perfect, so that a small fraction of the wave is still reflected. Ideally, the distance of vertical boundaries with respect

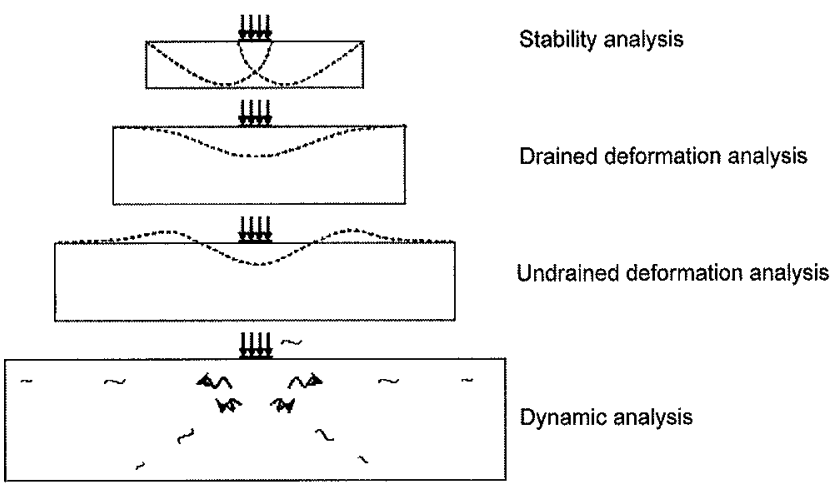

flc 4 Selection of vertical boundaries.

to the dynamic source should be more than half the wave velocity times the time interval of the calculation, such that reflected waves do not interfere with the dynamic source.

Regarding the position of the bottom boundary, one should consider the relative change of stress, taking into account the spreading of stress changes with depth. The bottom boundary should be put at the depth where the relative stress change is less than 10\% as illustrated in figure 5 (see also Vermeer \& Wehnert, 2005). The use of a small-strain stiffness model can help to reduce the dependency of results on the position of the bottom boundary (Brinkgreve et al., 2006).

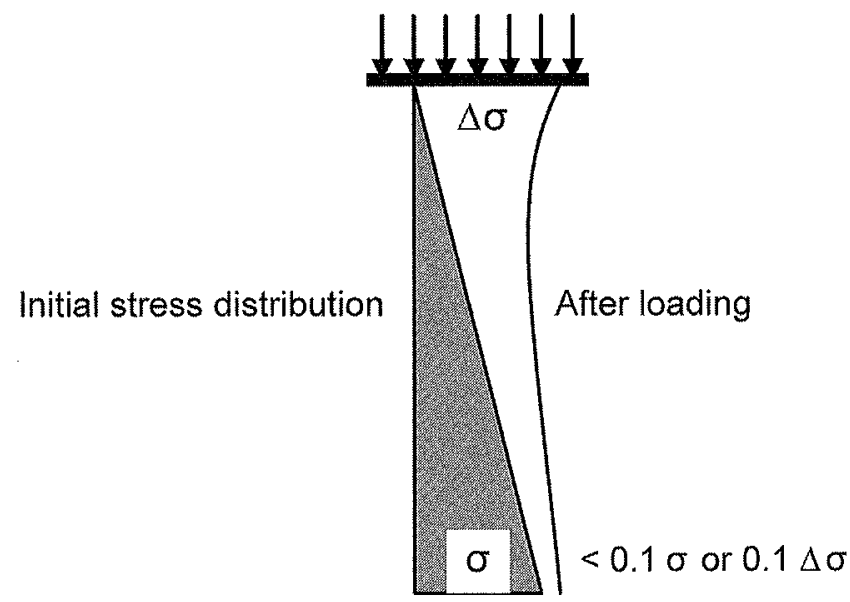

Selection of bottom boundary based on relative stress criterion. 


\section{Structural elements}

Structures in geotechnical applications, like piles, walls, anchors, plates, tunnel linings, etc., are often slender, which means that their thickness is small compared to their length making them well-adapted for being modelled by means of beam or plate elements. Morevover, using volume elements to model such structures could lead to badly shaped elements with unacceptably large aspect ratio. In most cases it is preferred to use beam elements for structures with a flexural rigidity. Such elements also have the advantage that the output of structural forces is directly available as primary output.

However, in the case of thick structures, volume elements with the properties of the corresponding material are more appropriate especially if transverse shearing or end-bearing capacity need to be accounted for within those structures. In order to be able to obtain structural forces, a very flexible fictitious beam may be placed inside the thick wall. This fictitious beam element should have the stiffness properties of the real structure divided by a high factor $\delta$ (in the order of $10^{6}$ ). When interpreting the structural forces, the results from the beam should be multiplied by the same factor $\delta$ in order to obtain realistic values. Note that this "trick" gives only realistic results in the case of elastic behaviour of the structure. This "trick" should not be used in the case of plastic structural behaviour or complex connections where typically $\mathrm{C} 1$-continuity (i.e. the continuity of the tangent along the neutral axis) is not satisfied between continuous structural members.

6ris.

\section{Interface elements}

Interfaces are used to model soil-structure interaction. Interfaces consist of node pairs with one node of the pair being connected to the structure and the other one being connected to the soil. Interface elements should primarily yield plastic deformations (and in this way reproduce possible sliding and gapping mechanism). Their strength is generally lower than the strength of the adjacent soil. The (non-physical) interface stiffness is generally large to avoid unrealistic elastic deformations. However, a stiffness that is too large can cause ill conditioning of the stiffness matrix, and should be avoided. Interfaces should be extended beyond the end of a structure or around corners (Fig. 6) to avoid locking and inaccurate stress results at the tip or corners of buried structures (Van Langen, 1991). Note that the strength of extended interfaces should not be reduced as it only represents a numerical artifact to improve stress calculation and has no reason to locally reduce the soil shear strength.
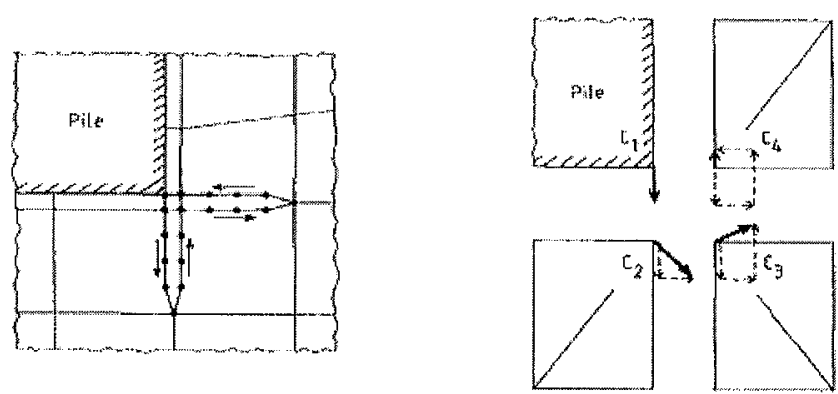

Extended interfaces around the corner of a structure (after Van Langen, 1991).
Care must be taken when using interface elements in plane strain models for situations that are actually not plane strain. For example, when modelling piles in a plane strain model by means of beam elements (see figure 3 for limitations) and using interface elements to model pile-soil interaction with reduced strength, it should be realized that the interface actually simulates an unrealistic sliding plane in the soil. It should be mentioned in this context that the use of interface elements with a reduced strength will probably lead to incorrect prediction of the skin resistance and it might be preferable not to use any interface at all for such situations. Optimally these types of problems are better addressed by 3D models.

\section{3}

\section{Material behaviour}

Soil can be regarded as a material with highly nonlinear properties. The behaviour of soil is described by constitutive models, ranging from the simple linearelastic perfectly plastic model to highly sophisticated non-linear anisotropic time-dependent models. Structures in the soil can often be regarded as stiff and elastic, but in some cases non-linear structural behaviour needs to be considered in more detail.

\section{1}

\section{Soil behaviour}

Before deciding which soil constitutive model to select, it is important to consider which features of soil behaviour are important in the situation at hand. A preliminary analysis with a simplified model could help to identify the dominant stress paths and stress levels. It is tempting to use simple models, since in general the number of model parameters is less than for advanced models. However, when dealing with various stress levels and stress paths in combination with a simple constitutive soil model, soil layers need to be divided into multiple sub-layers, each of them having their own set of model parameters. Advanced models may include additional features like stress-stress path- or strain-dependency of stiffness or anisotropy. Therefore, the effort of determining more parameters may be less than the total effort of determining different sets of model parameters for a simple model. For more details about the possibilities and limitations of soil models and parameter selection (see Brinkgreve, 2005).

\section{9}

\section{Undrained behaviour}

A particular type of soil behaviour that needs special attention is undrained behaviour. Undrained behaviour can be modelled in different ways, each of them having their possibilities and limitations. The classical approach of modelling undrained soil behaviour is by means of a total stress analysis (Fig. 7a). In this case, undrained values for stiffness and strength parameters $\left(E_{u,}, v_{u}, s_{u}\right.$ and $\left.\varphi=0\right)$ must be given as model input. This method is still rather popular amongst the geotechnical FE modelling community who finds it convenient at several design stages to directly input the shear strength values. The disadvantage of this method is 
that no distinction is made between pore pressures and effective stresses, which makes it impossible to perform a subsequent consolidation analysis. Note moreover that the shear strength needs to be changed in the case of a change of effective stress, for example as a result of consolidation.

Undrained behaviour can also be modelled by an effective stress approach using effective stiffness parameters $\left(E^{\prime}\right.$ and $\left.v^{\prime}\right)$, while adding a separate large bulk stiffness for the pore water $\left(\mathrm{K}_{\mathrm{w}} / \mathrm{h}=\right.$ high) (Fig. $7 \mathrm{~b}$ and c). In this way, distinction can be made between effective stresses (based on the stiffness of the soil skeleton) and excess pore pressures (based on the stiffness of the pore water). The actual development of pore pressures depends on the selected soil constitutive model. In principle, soil strength can be modelled using the Mohr-Coulomb criterion with effective strength properties, provided that the soil model predicts the right pore pressures. The obtained undrained shear strength is a result of the effective stress path (ESP). In particular for soft soils, most constitutive models do not predict the right pore pressures and over-predict the undrained shear strength when using this approach. Therefore, resulting stress states should always be checked against a known shear strength profile. In the case that the resulting shear strength is not modelled properly, an alternative effective stress approach can be used in which the strength is modelled using a Tresca criterion with direct input of the undrained shear strength $s_{u}$ (Fig. $7 d$ ). Note that in this case, the shear strength would need to be changed in the case of a change of effective stress. It is also important to realize that this alternative method often leads to incorrect prediction of the excess pore pressures, but this does not have consequences for the soil strength and stability.

\section{Meshing}

In the finite element method, the model geometry is divided into finite elements, which is called a mesh. The accuracy of the finite element model depends on the number of elements used and the order of interpolation of the primary variables within the elements. When low-order elements and/or an insufficiently number of nodes are used, the flexibility in the mesh is insufficient such that deformations are under-estimated and failure loads are over-estimated. For serviceability states in geotechnical applications the use of quadratic or higher order elements is recommended to avoid mesh locking problems. For ultimate limit state calculations quadratic elements may still overestimate failure loads; therefore higher order elements are preferred for such situations. Locally finer meshes (smaller elements) need to be used at locations where concentrations of stress, strain or flow is expected, such as near structures and loads (Fig. 8). Even when using high-order elements, structural sections should be modelled with more than just one element. Coarser meshes may be used towards the model boundaries since lower stress gradients are expected to develop there.

In the case of dynamic calculations, there are special requirements for the size of elements, related to the range of frequencies that need to be modelled accurately. The wave length of a shear wave $L_{s}$ is defined as the shear wave velocity $v_{s}$ over the frequency $f$. The shear wave velocity $v_{s}$ is a function of the shear stiffness $G$ and the material density $\rho: v_{s}=\sqrt{ }(G / \rho)$. For quadratic elements, the wave length should be described by at least 8 elements.

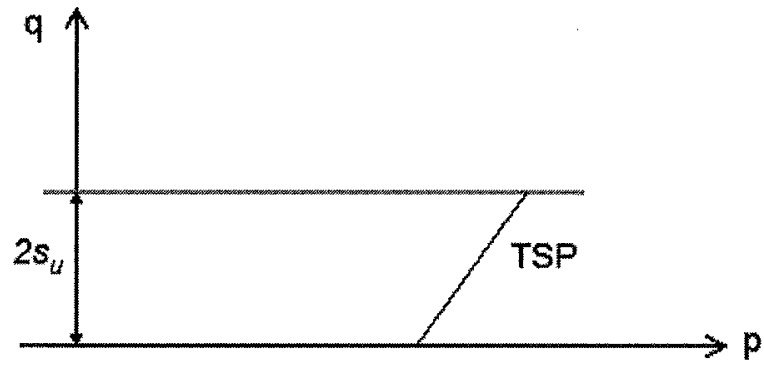

a. Undrained total stress approach

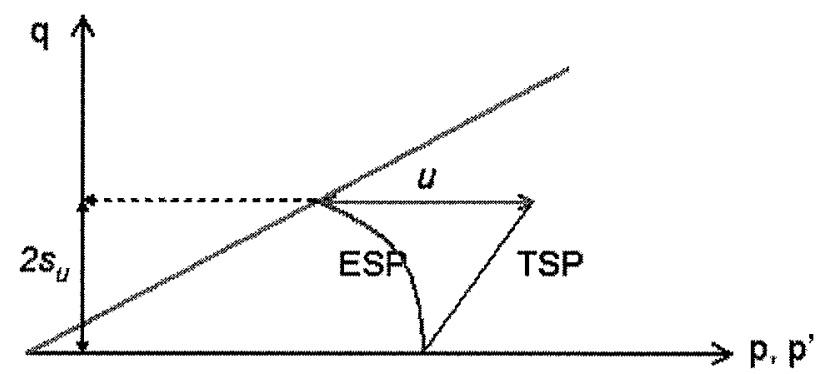

c. Undrained effective stress approach: advanced model

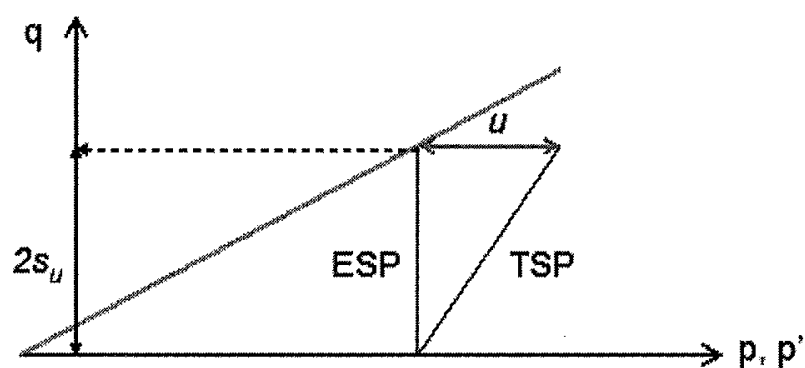

b. Undrained effective stress approach: simple Mohr-Coulomb model

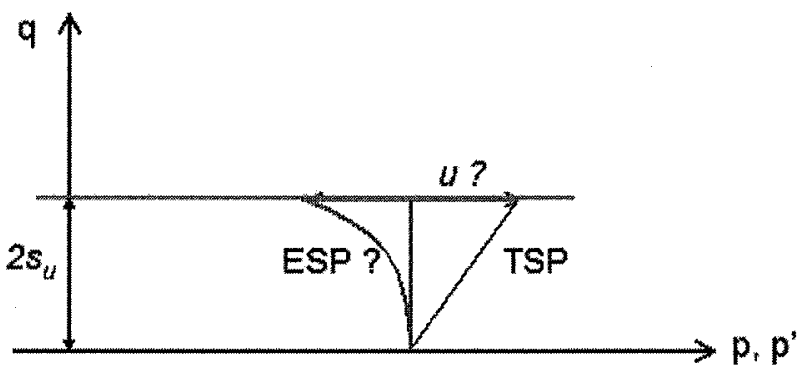

d. Undrained effective stress approach with Tresca model

176. 7 Different ways to model undrained behaviour. 

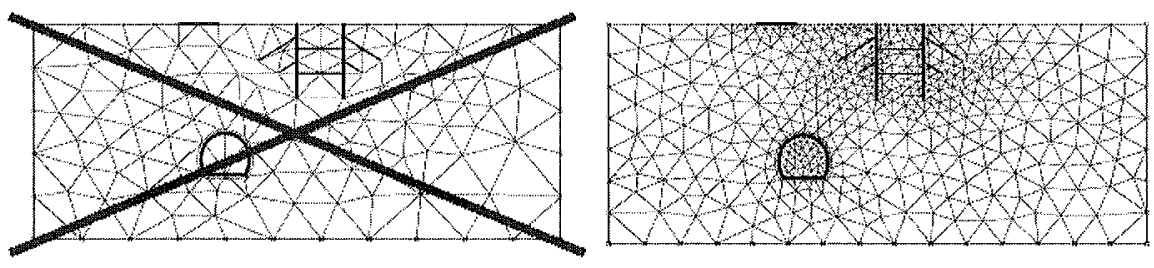

HG a Different ways to model undrained behaviour.

\section{5}

\section{Initial conditions}

In reality, initial stresses and conditions are present in the soil, which are of major influence on a new structure to be built in the ground. The finite element model does not know of any initial conditions unless this is explicitly generated. The initial conditions involve at least initial stresses, preferably with a distinction between pore pressures and effective stresses. Depending on the type of soil constitutive model used, initial values of state parameters may need to be set as well, such as void ratio or pre-consolidation stress.

\section{$5 \pi$}

\section{Initial stresses}

Initial stresses in the ground may be introduced in different ways. A simple procedure makes use of the soil total weights of the soil layers to generate total vertical stresses. Subtracting the pore pressure gives the effective vertical stress, whilst multiplying this vertical effective stress by a specified coefficient of lateral stress $\mathrm{K}_{0}$ gives the horizontal effective stress. This socalled $\mathrm{K}_{0}$-procedure does not guarantee equilibrium to be satisfied, but is suitable in situations with only horizontal soil layers. Using the $\mathrm{K}_{0}$-procedure in situations involving slopes or non-horizontal layers (Fig. 9) leads to an initial unbalance (Fig. 10), which needs to be equilibrated before performing any further loading or construction stages.

A possible alternative to the $\mathrm{K}_{0}$-procedure is the use of a true finite element procedure to generate initial stresses due to soil weight, is gravity loading. In this case equilibrium is guaranteed within the tolerances of the finite element calculation. Lateral soil stresses are now the result of the parameters used in the soil constitutive model. For a simple linear-elastic perfectly-plastic model in the case of one-dimensional compression (Figure 9a), the resulting coefficient of lateral stress is found to be $K_{0}=v /(1-v)$, where $v$ is Poisson's ratio. The disadvantage of using gravity loading in one calculation is that $\mathrm{K}_{0}$ cannot be greater than unity (1.0), since Poisson's ratio should be lower than one half $(0.5)$. To overcome this limitation, for example for over-consolidated soils that might have a higher $\mathrm{K}_{0}$-value, gravity loading should first be performed with a high Poisson's
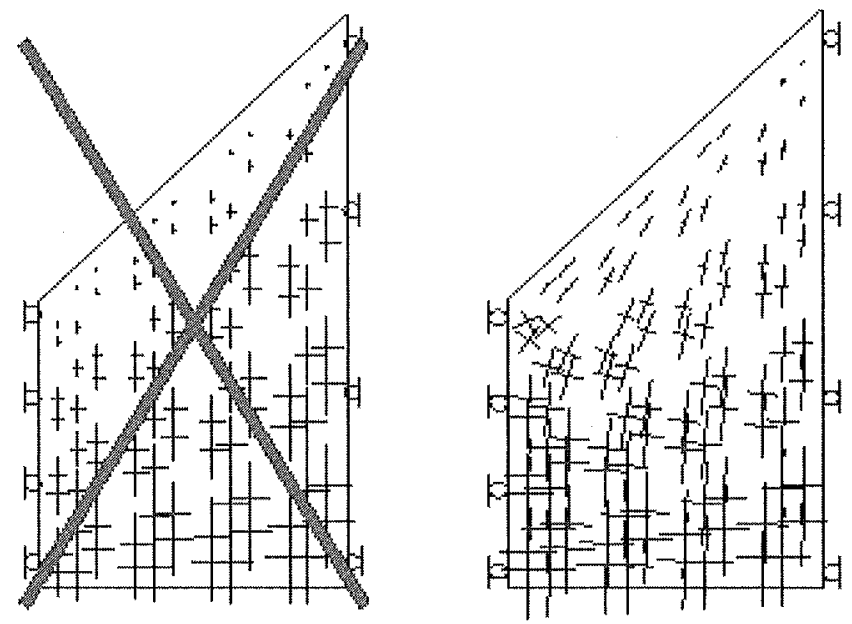
$\mathrm{K}_{0}$-proce
slope.

ratio to a higher level of gravity after which unloading to normal gravity should be performed using a low Poisson's ratio. Poisson's ratio may again be changed for subsequent calculations.

Initial stresses may also be influenced by previous constructions in the neighbourhood. When this is the case, these constructions need to be simulated first as part of the initial stress generation, before the actual project is considered.

Note finally that when generating initial stresses, the soil should be modelled as a drained material; otherwise unrealistic initial stresses and pore pressures are created.

\section{2}

\section{Initial pore pressures}

Pore pressures also play an important role in the soil. Especially in excavations, pore pressures can form a major part of the loads on soil retaining structures, since their lateral load component is equal to the vertical component. Therefore, it is important to model pore pressures appropriately.

A simple way to model (initial) pore pressures is to use a phreatic level and to generate hydrostatic pore
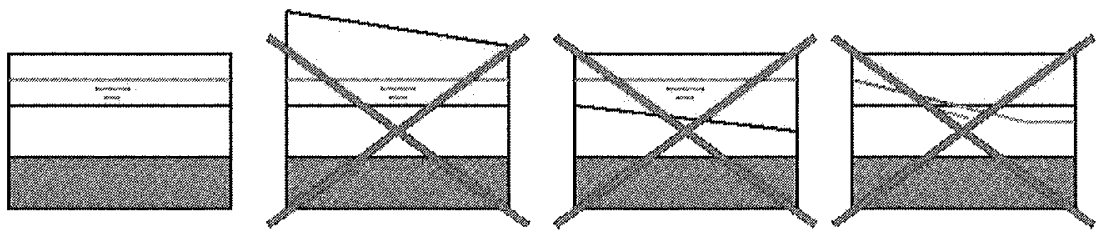

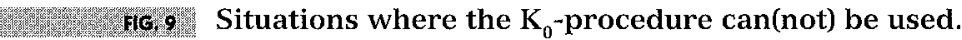


pressures under this level. Care has to be taken when the phreatic level is not horizontal. This is equivalent to a groundwater head which is not constant, as a result of which groundwater flow will occur, which may result in a non-hydrostatic pore pressure distribution.

\section{Initial values of state parameters}

Advanced models generally have parameters to describe the history or condition of the soil, such as the pre-consolidation stress, the void ratio, the relative density or the state of anisotropy. Together with the generation of initial stresses, these so-called state parameters need to be initialized as well, based on additional information. In the case of gravity loading, the history should be simulated as realistic as possible. Also here, the $\mathrm{K}_{0}$-procedure has limitations in the sense that it does not allow for a true simulation of the history, as a result of which the initial value of state parameters might be inaccurate.

\section{6}

\section{Calculations}

After the finite element model has been created and the initial conditions have been generated, the calculations need to be defined. A finite element analysis can consist of multiple calculation phases, similar to construction stages in a real project.

When dealing with structures in the soil, it must be realized that the construction process in reality involves installation effects, and it should be considered whether it is important and how it can be included in the finite element model. In most cases structures are simply ( wished in place ). In that case it must be considered what the consequences are of this simplification.

Similar as what has been mentioned for the generation of initial stresses, pore pressures can have a significant influence on the results. Therefore, care must be taken with the definition of hydraulic conditions (see figure 11). In the case of a groundwater flow calculation in a symmetric model of which only one half is modelled, flow through the symmetry boundary must be prevented, so the boundary must be "closed". The same applies to coupled consolidation calculations. Always check the pore pressure distribution for unexpected "jumps" in pore pressures.

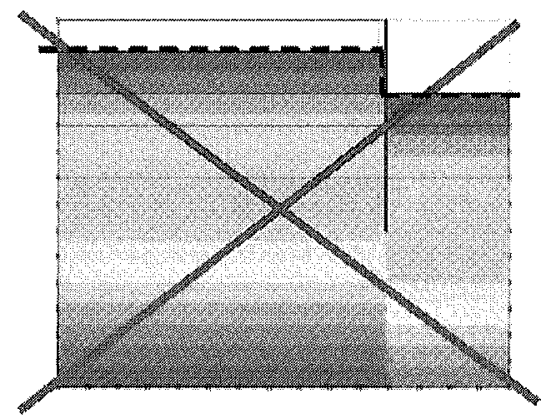

a)
A typical situation where this might occur is a dry excavation in permeable soil. Figure $12 \mathrm{a}$ shows the wrong use of a phreatic level with a jump. Note that the jump leads to a difference in pore pressures all the way to the bottom of the model. In such a case the use of a local pore pressure distribution should be considered. An even better solution would be to perform a groundwater flow calculation to generate the (initial) pore pressure distribution (Fig. 11b). This requires the proper use of permeabilities and hydraulic conditions at the model boundaries. In this case a groundwater head is specified at both vertical model boundaries and at the excavation bottom, whereas the bottom boundary is closed.

For calculations that involve a physical time step, conditions may apply with respect to the minimum or maximum time step that can be taken in the calculation. Coupled consolidation analysis requires a time step larger than a particular minimum time step in order to avoid stress oscillations (Vermeer \& Verruijt, 1981). Dynamic calculations require a time step smaller than a particular maximum time step in order to ensure that waves do not travel more than one element per time step.

When performing safety analysis by means of strength reduction (phi-c reduction) it should be realized that a safety factor can only be obtained as soon as a full mechanism has developed. This requires a sufficient number of calculation steps to be performed. If the calculation stops before a full mechanism has developed, the calculation should be repeated with a larger number of steps, until the calculation control parameter is "over the top" when plotting it against the displacement of a point within the moving soil mass (Fig. 12). It should be realized that the displacements themselves are not realistic. In order to view the developed mechanism, attention should be focused on the plastic deformations or the displacement increments rather than the total displacements. It is also important to point out that safety factors computed in the framework of a phi-c reduction analysis have a different meaning than those generally considered by structural engineers and defined as the ratio of the failure load over the working load.

Proper attention should also be paid to the way structural members are being modelled during phi-c reduction analyses. Indeed assuming elastic behaviour for the structural members might have consequences on the resulting safety factor. It is therefore advised to carefully monitor the evolution of the structural forces and moments during the entire phi-c reduction calcu-

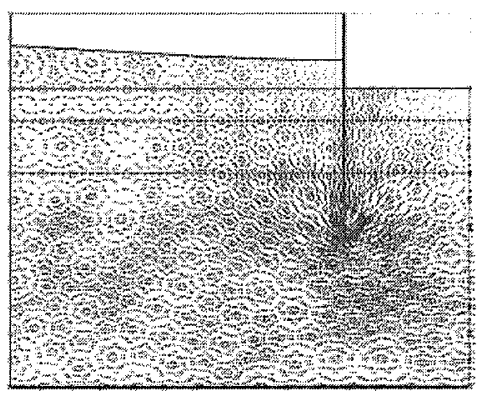

b)

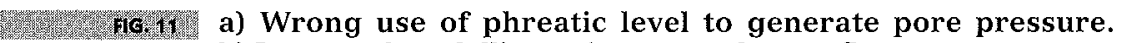
b) Improved modelling using groundwater flow. 


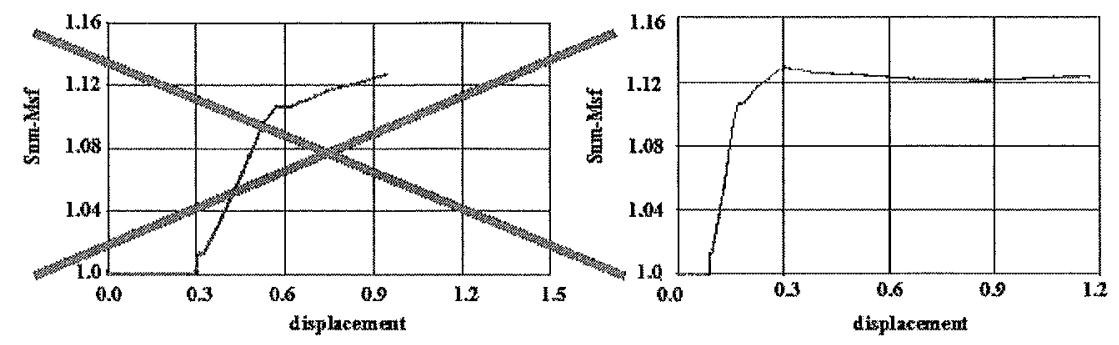

14. 12. 12 Safety factor can only be obtained when a full mechanism has developed.

lation process. Finally it is also important to mention that considering or ignoring the soil undrained beha* viour might have non-negligible influence on the resulting safety factor depending mostly whether the effect of generation of excess pore pressures has a positive or negative effect onto the mobilized friction.

\section{Numerical procedures}

Geotechnical finite element calculations are divided into steps to deal with the non-linearity of the soil behaviour. Different numerical solution algorithms exist to solve the sets of constitutive equations produced the FE discretization. Care must be taken with explicit schemes which are conditionally stable and might excessively deviate from equilibrium or even become unstable. Nowadays, most numerical solution algorithms are based on an implicit integration scheme and involve an iteration procedure to satisfy equilibrium within a certain tolerance for each loading step (also referred to as a Newton-Raphson procedure). Such procedures use an error criterion to check whether a solution has converged. It is tempting to start playing with the iterative procedure settings in an attempt to speed up the calculation process or ease a slow and difficult convergence process. In this context, manipulating those advanced settings and particularly increasing the tolerated error must be done with great care as results might become simply unreliable since equilibrium will not be properly enforced. This is well illustrated in figure 13 where two analyses of the same dry excavation model have been carried out using different tolerated errors of respectively $1 \%$ (which is a rather standard default value) and $20 \%$. One can clearly see that the computed wall settlement is twice as large using the $1 \%$ error compared to the value obtained with a $20 \%$ error.

\section{7}

\section{Interpretation of results}

When all modelling issues have been properly taken into account and the calculation has run successfully, there is no guarantee that the finite element results are correct and can directly be used in geotechnical design. The user should be aware that a converged solution does not mean that he is dealing with a reliable result. First, the results of each individual calculation phase need to be inspected carefully, with a critical attitude. Actually, the user should have a clear expectation of the results on the basis of his experience before inspecting the outcome of the calculations. The

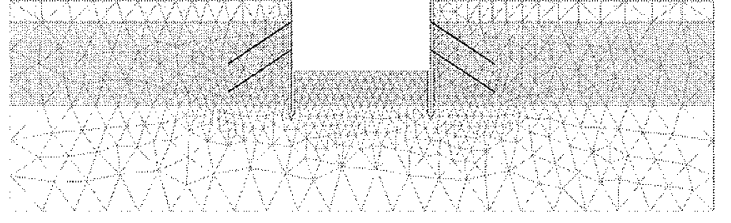

a) Mesh overview

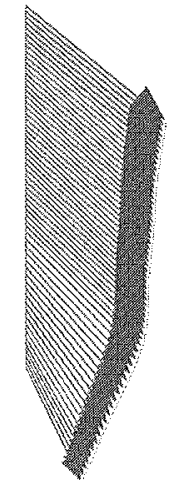

umax $=42.2 \mathrm{~mm}$

b) Wall displacement with $1 \%$ error

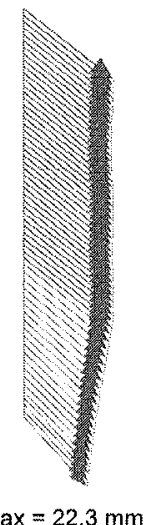

c) Wall displacement with $20 \%$ error
Hered of tolerated error on wall displacement for a dry excavation model.

more output features are reviewed the better. When the calculated results show peculiarities or differ from the expected results, a consistent explanation needs to be found. Results that cannot be explained should not be accepted.

When the results have been verified and found to be consistent, an interpretation and translation can be made towards the geotechnical design issues. Thereby the consequences of simplifications and limitations in the finite element model and the selected constitutive model need to be taken into consideration. Are the results realistic, conservative or optimistic?

\section{8}

\section{Visual checklist}

A basic requirement for a satisfactory finite element calculation is that the used finite element software is reliable. This requires at least that the software has been verified. Verification in this respect means a check whether the software is able to reproduce known solutions. This is usually done by the software developer, but it does not harm if users of the software redo some of the verification examples or check others. Successful verification does not automatically mean that 
the software will produce realistic results in practical applications. The latter is more related to validation rather than verification. Validation in this respect is a procedure to demonstrate that the outcome of a calculation matches reality to a great extend; for example based on in-situ measurements. Validation, therefore, is not only related to the reliability of the software, but also to the ability of the user to make a good finite element model.

To help geotechnical engineers to make reliable finite element models, a checklist is being developed at Plaxis containing items such as described in this publication to make the engineers aware of the traps and pitfalls. The checklist is supported by visual examples, such that they could easily be recognized. Complying with all items in the checklist does not mean that the finite element model automatically gives good results. Engineering judgment is always required to interpret and translate the results into geotechnical design issues. This remains the responsibility of the engineer and the professional environment around him.

\section{Conclusions}

The finite element method is a great tool for the analysis and design of geotechnical engineering applications. However, the accuracy of finite element calculations depends on the ability of the user of the finite element software to deal with the possibilities and limitations of the various models and methods used. In this publication, several traps and pitfalls that could lead to incorrect results if they are not recognized and understood have been described. To help engineers to make reliable finite element models, a visual checklist is being developed by Plaxis. Despite the availability of such tools, the engineer remains responsible for the translation of results into geotechnical design issues.

\section{Bibliographie}

Brinkgreve R.B.J. - Selection of soil models and parameters for geotechnical engineering applications. Geofrontiers (ed. Jamamuro), ASCE, 2005.

Brinkgreve R.B.J., Bakker K.J., Bonnier P.G. - The relevance of small-strain stiffness in numerical simulation of excavation and tunnelling projects. NUMGE (ed. Schweiger). Taylor \& Francis - London, 2006, p. 133-139.

Brinkgreve R.B.J., Swolfs W.M., Engin E. Plaxis user manual. Plaxis bv, Delft, 2010.
Meißner H. - Baugruben - Empfehlungen des Arbeitskreises 1.6 "Numerik in der Geotechnik", Abschnitt 3, Geotechnik 25, 2002, p. 44-46.

Van Langen H. - Numerical Analysis of Soil-Structure Interaction. Dissertation. Delft University of Technology, 1991.

Potts D.M., Zdravkovic L. - Finite Element Analysis in Geotechnical Engineering: Application. Thomas Telfort, London, 2001.
Vermeer P.A., Verruijt A. - An accuracy condition for consolidation by finite elements. Int. J. for Num. Anal. Meth. in Geom., 5, 1981, p. 1-14.

Vermeer P.A., Wehnert M. - Beispiele von FE-Anwendungen - Man lernt nie aus. FEM in der Geotechnik (ed. Grabe et.al.). Technische Universität HamburgHarburg, 2005. 